\title{
DC MAGNETIC FIELD GENERATOR WITH SPATIAL COILS ARRANGEMENT
}

\author{
Adam Kozlowski, Stan Zurek ${ }^{2}$ \\ ${ }^{I}$ Magneto Ltd., Odlewnikow 43, 42-200 Czestochowa, Poland \\ ${ }^{2}$ Megger Instruments Ltd, Archcliffe Road, Dover, CT17 9EN, United Kingdom \\ adam.kozlowski@magneto.pl,stan.zurek@megger.com
}

\begin{abstract}
This paper is dedicated to the effect of the number of excitation coils of a DC magnetic field generator with magnetic core on uniformity of flux density in an air gap. The advantages and drawbacks of the air gap surrounded by yoke elements with coils were analysed.
\end{abstract}

\section{Introduction}

Many industrial or laboratory research processes require the presence of a magnetic field, for formation or measurement of magnetic properties.

Permanent magnets are an easily accessible source of a magnetic field. Especially widespread are neodymium magnets characterized by large values of energy densities.

Just a single magnet is in fact a generator of a constant (DC) magnetic field. One can make a more complicated construction of a magnetic field generator (MFG) with a few magnets as well as from magnets combined with a magnetic core to increase the magnetic field strength in the volume of interest - often an air gap. The main benefit of MFG based on magnets is the generation of a magnetic field without an energy supply. The drawback is the lack of possibility to regulate and/or switch off the magnetic field.

Another MFG construction is a magnetic core with an air gap and excitation windings or coils as sources of magnetomotive force (MMF). This solution enables full control of magnetic field strength in the air gap between two poles of the magnetic circuit.

There are many configurations of electromagnetic field generators (EMFG). They differ by arrangement of excitation coils, their quantity and core shape, and so on. As a novel design could be considered an EMFG characterized by a more complex core design - with coil arrangement spatially. An analysis of results was conducted on the basis of measurements carried out on a test model of such an EMFG with spatial coils arrangement in order to investigate usefulness of this kind of generator for industrial and laboratory applications. 


\section{Types of magnetic field generators. Laws related to magnetic phenomenon}

In the case of the type of excitation applied, MFG can be divided into magnetic and electromagnetic devices. Both mentioned types can be coreless and with a magnetic core, which is a low reluctance path for magnetic flux.

Coreless MFG is nothing else than just a permanent magnet, generating a magnetic field around itself. Placing two magnets with their opposite poles at some distance from each other results in accordingly higher magnetic field strength in the air gap between them (Fig. 1a, b). Combining two magnets with a magnetic core made for instance from iron, magnetic field in the air gap will be stronger still (Fig. 1c).

Magnetic core is a low reluctance path for magnetic flux, as compared to the air gap. This results directly from the values of relative magnetic permeability of the ferromagnetic material and the air.

a)

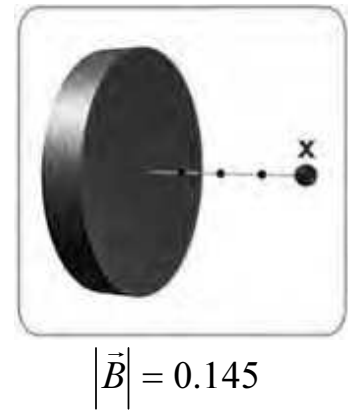

b)

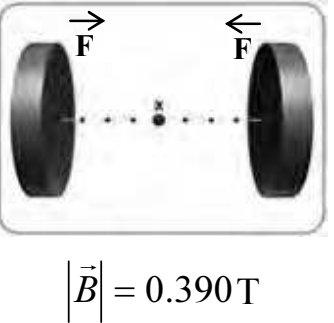

c)

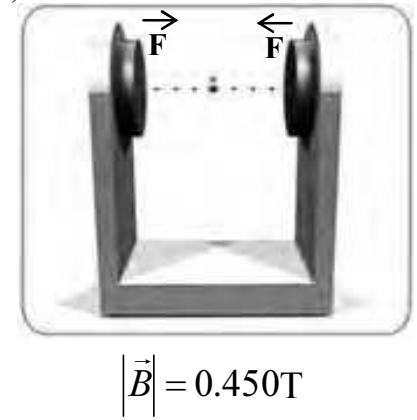

Fig. 1. Magnetic flux density $|\vec{B}|$ of cylindrical magnets N42 $50 \times 20 \mathrm{~mm}$ in distance $\mathrm{x}=20 \mathrm{~mm}$ [1]: a) from pole of one magnet, $\mathrm{b}$ ) between poles of two magnets,

c) between poles of two magnets combined with a magnetic core

The electrical coil is another type of excitation. The relationship between the magnetic field and the electrical current are determined by Biot-Savart and Ampere laws.

Biot-Savart law determines the induction of magnetic field, comes from a small section $d \vec{l}$ of rectilinear electrical conductor with current $I$, in the specified point of area (Fig. 2).

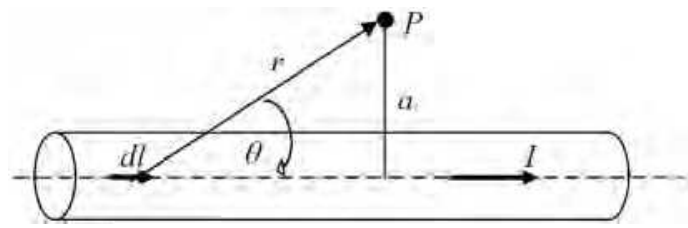

Fig. 2. Rectilinear electrical conductor with current 


$$
\vec{B}=\frac{\mu_{0}}{4 \pi} \cdot \int \frac{I d \vec{l} \times \hat{\vec{r}}}{r^{2}}
$$

By integrating the above formula over infinite length of the conductor a new formula is obtained, for the magnetic induction $|\vec{B}|$ from the distance $a$ from the rectilinear conductor axis conducting the electrical current

$$
|\vec{B}|=\frac{\mu_{0} I}{2 \pi a}
$$

where $\mu_{0}$ - absolute magnetic permeability of vacuum: $4 \cdot \pi \cdot 10^{-7} \mathrm{H} / \mathrm{m}$.

The value of the magnetic field around the rectilinear conductor with current (Fig. 3) can be also defined from the Ampere's law,

$$
\oint \vec{B} \cdot d \vec{l}=\mu_{0} I
$$

and after some transformations

$$
\vec{B} \oint d \vec{l}=B 2 \pi a=\mu_{0} I
$$

the same formula of magnetic induction like (2) - from Biot-Savart law - is obtained.

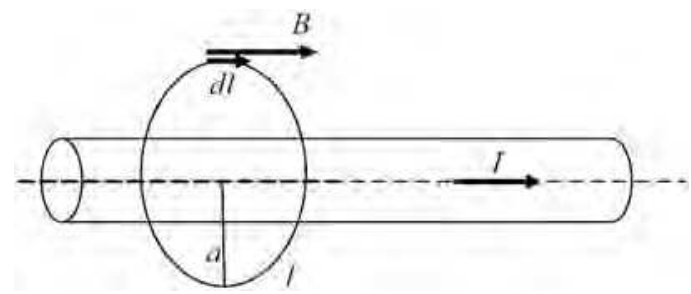

Fig. 3. Rectilinear conductor with current surrounded by a flat contour $l$ in area perpendicular to the conductor

From the mathematical point of view, the Ampere law is the equation of magnetic vector $\vec{B}$ circulation as integral $\oint \vec{B} \cdot d \vec{l}$ along a flat closed contour $l$. law

Magnetic field strength generated by an electrical coil is determined by Ampere

$$
\oint_{C} \vec{H} \cdot d \vec{l}=I
$$

where:

$I$ - electrical current flowing through any area stretched on the closed contour $C, \mathrm{~A}$, $\vec{H}$ - vector of magnetic field strength, A/m. 
Magnetic field strength $\vec{H}$ does not depend on magnetic features of the environment, but on the other hand the magnetic flux density $\vec{B}$ does. Both values are defined interchangeably for the magnetic field and also concerns the magnetic field generating in the air gap of the magnetic field generator.

The relationship between magnetic flux density $|\vec{B}|$ and magnetic field strength $|\vec{H}|$ in a scalar notation is as follows:

$$
|\vec{B}|=\mu_{0} \cdot \mu_{r} \cdot|\vec{H}|
$$

where:

$\mu_{0}$ - absolute magnetic permeability of vacuum: $4 \cdot \pi \cdot 10^{-7} \mathrm{H} / \mathrm{m}$,

$\mu_{r}$ - relative magnetic permeability of environment; for air it can be assumed that $\mu_{r}=1$,

$|\vec{B}|$ - absolute value of magnetic induction, T,

$|\vec{H}|$ - absolute value of magnetic field strength, $\mathrm{A} / \mathrm{m}$.

Using the equation (6), the formula (2) can be shown as:

$$
|\vec{H}|=\frac{I}{2 \pi a}
$$

An electrical coil without a magnetic core, often referred to as solenoid, generates a magnetic field inside and around itself (Fig. 4). The value of the magnetic field strength in geometrical centre of the solenoid, with a length ten times greater than the diameter, can be described by formula (8) with good approximation. The further from the geometrical centre, the lower the magnetic field strength. Compared to the centre, at the ends of the solenoid, the value of magnetic field strength decreases roughly by half. The theoretical meaning of an "ideal" solenoid is an infinitely long coil.

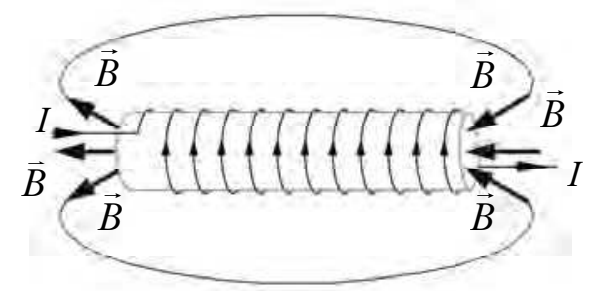

Fig. 4. Solenoid - electrical coil without magnetic core: $I$ - electric current in coil winding, $\vec{B}$ - vector of magnetic flux density generated inside and around of solenoid in air 


$$
|\vec{H}|=\frac{N \cdot I}{|\vec{l}|}
$$

where:

$|\vec{H}|$ - absolute value of magnetic field strength, $\mathrm{A} / \mathrm{m}$,

$N$ - number of turns of the coil,

$I$ - electric current in the coil, A,

$|\vec{l}|$ - absolute value of length of solenoid, $\mathrm{m}$.

Considering the solenoid with magnetic material (magnetic core) inside, the part of the magnetic path is characterized by lower reluctance compared to the other part of magnetic circuit in the air. If the magnetic core is shaped appropriately, the lines of magnetic flux will be arranged in a required way.

A device containing a magnetic excitation in the form of an electrical coil, properly shaped magnetic core made of ferromagnetic material can be named as electromagnetic generator of magnetic field (EMFG). The required field is generated in an air gap between the two ends (poles) of the core, or the magnetic circuit (Fig. 5).

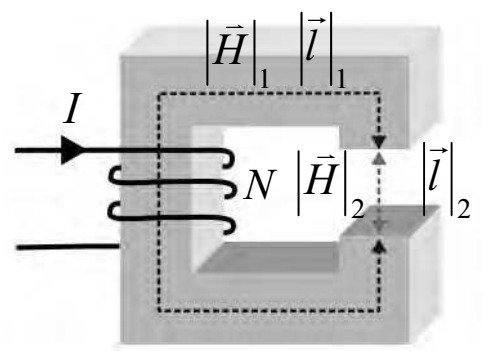

Fig. 5. Electromagnetic core generator of magnetic field: $N$ - number of turns of the coil, $I$ - electric current in the coil [A], $|\vec{H}|_{1}$ - absolute value of magnetic field strength in magnetic core [A/m], $|\vec{H}|_{2}$ - absolute value of magnetic field strength in air gap [A/m], $|\vec{l}|_{1}$ - absolute value of length of magnetic path [m], $|\vec{l}|_{2}$ - absolute value of length of air gap [m]

Inside a long solenoid with magnetic excitation or magnetomotive force equal to $N \cdot I$, the magnetic field strength can be calculated from the formula (8), considering $|\vec{l}|$ as the length of solenoid. For the magnetic field generator from Figure 5 with the same excitation $N \cdot I$, the magnetic field strength in the air gap can be calculated using the same formula, but $|\vec{l}|$ should be considered as the length of an air gap $|\vec{l}|_{2}$ instead of the coil length. This occurs, when the product $\left|\vec{H}_{1}\right| \cdot|\vec{l}|_{1}$ (for the magnetic core) can be neglected in the Ampere's law: 


$$
N \cdot I=|\vec{H}|_{1} \cdot|\vec{l}|_{1}+|\vec{H}|_{2} \cdot|\vec{l}|_{2}
$$

In typical core arrangements of EMFG the reluctance of the gap is much greater than that of the core, so that the contribution from the core can be neglected, especially for circuits for which flux fringing is not severe, and the working point does not approach saturation of the core.

Taking into account shape, magnetic cores can be divided into simple and multi-path, and the latter ones can be further subdivided into symmetrical and asymmetrical cores.

\section{Magnetic field generator with spatial coils and side yokes}

EMFG with spatial coils arrangement (Fig. 6) is characterized by modular construction, enabling convenient control over:

- shape and dimensions of poles as well as length of the air gap,

- number of coils and side yokes,

- different configuration of coils connection.

a)

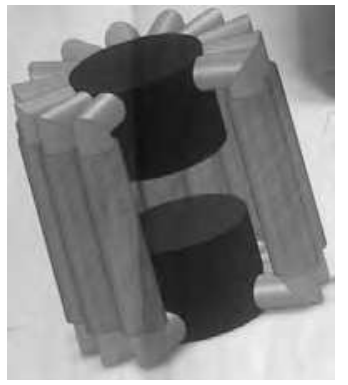

b)

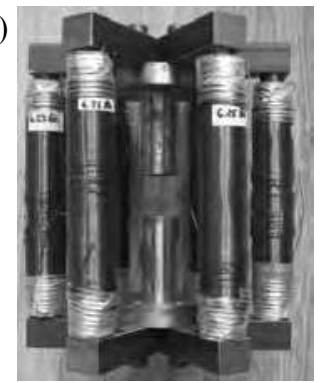

Fig. 6. Magnetic field generator with spatial coils arrangement: a) conceptional model; b) test model

The main concept behind producing the test model was to investigate the influence of the side yokes and coils presence around the air gap on the uniformity of the magnetic field generated in the air gap. The project aimed to generate magnetic flux density $|\vec{B}|=100 \mathrm{mT}$ in the air gap with length $|\vec{l}|=20 \mathrm{~mm}$. The value of magnetic flux density corresponds to magnetic field strength $|\vec{H}|=79.6 \mathrm{kA} / \mathrm{m}$ formula (6). The required excitation is therefore $N \cdot I=1884$ ampere-turns.

\section{Influence of spatial coils arrangement on the uniformity of magnetic field in the air gap}

To examine the influence of spatial coils arrangement on the uniformity of magnetic field in the air gap, two main configurations of the magnetic field genera- 
tor were set up, with various modifications. In configuration 1 (Fig. 7a) - the number of side yokes with coils was changed symmetrically from 2 to 6 pieces (Fig. 6b). In configuration 2 (Fig. 7b) only the number of side yokes was changed from 2 to 6 , whereas two coils were installed on the two pole pieces. Because of different length of poles to the length of side yokes, excitation coils from configuration 2 have different dimensions comparing to the coils from configuration 1, but the excitation (MMF) was the same in both cases during the experiments.

Specification of EMFG configurations and their modifications are shown in Table 1.

a)

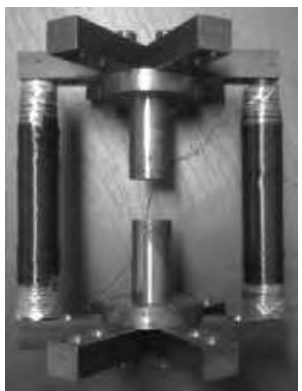

b)

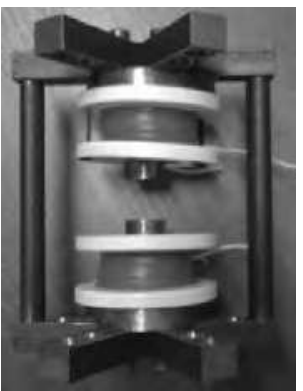

Fig. 7. Test model of magnetic field generator: a) configuration 1, b) configuration 2

Table 1

Specification of magnetic field generator configurations and modifications

\begin{tabular}{|c|c|c|}
\hline & Configuration 1 & Configuration 2 \\
\hline Diameter of pole pieces & $20 \mathrm{~mm}$ & $20 \mathrm{~mm}$ \\
\hline Shape of pole pieces & cylindrical & cylindrical \\
\hline Diameter of side yokes & $12 \mathrm{~mm}$ & $12 \mathrm{~mm}$ \\
\hline Number of side yokes & $2 \div 6$ & $2 \div 6$ \\
\hline Number of coils & $2 \div 6$ & 2 \\
\hline Internal diameter of coil & $15 \mathrm{~mm}$ & $30 \mathrm{~mm}$ \\
\hline Length of coil & $110 \mathrm{~mm}$ & $20 \mathrm{~mm}$ \\
\hline Number of turns of each coil & 1000 & 500 \\
\hline Location of coils & side yokes & poles \\
\hline
\end{tabular}

Graphs of the magnetic flux density vs. magnetomotive force (excitation) is shown in Figures 8a and 9a. The magnetic flux density was measured along the symmetry axis from pole to pole, and two values - minimum and maximum - are also shown on the charts.

The percentage difference between these maximum and minimum values (Figs $8 \mathrm{~b}$ and $9 \mathrm{~b}$ ) can be used as a measured of non-uniformity of the flux density distribution, and is calculated as follows:

$$
D=\frac{|\vec{B}|_{\max }-|\vec{B}|_{\min }}{|\vec{B}|_{\min }} \cdot 100, \%
$$


a)

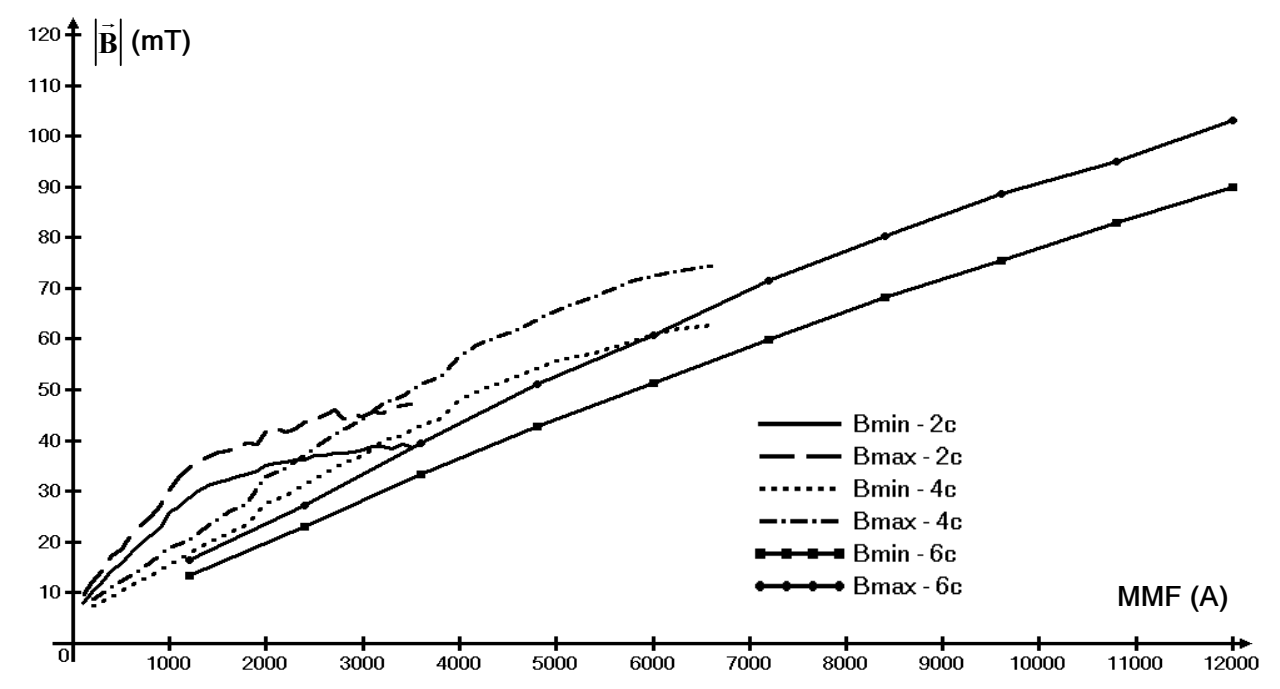

b)

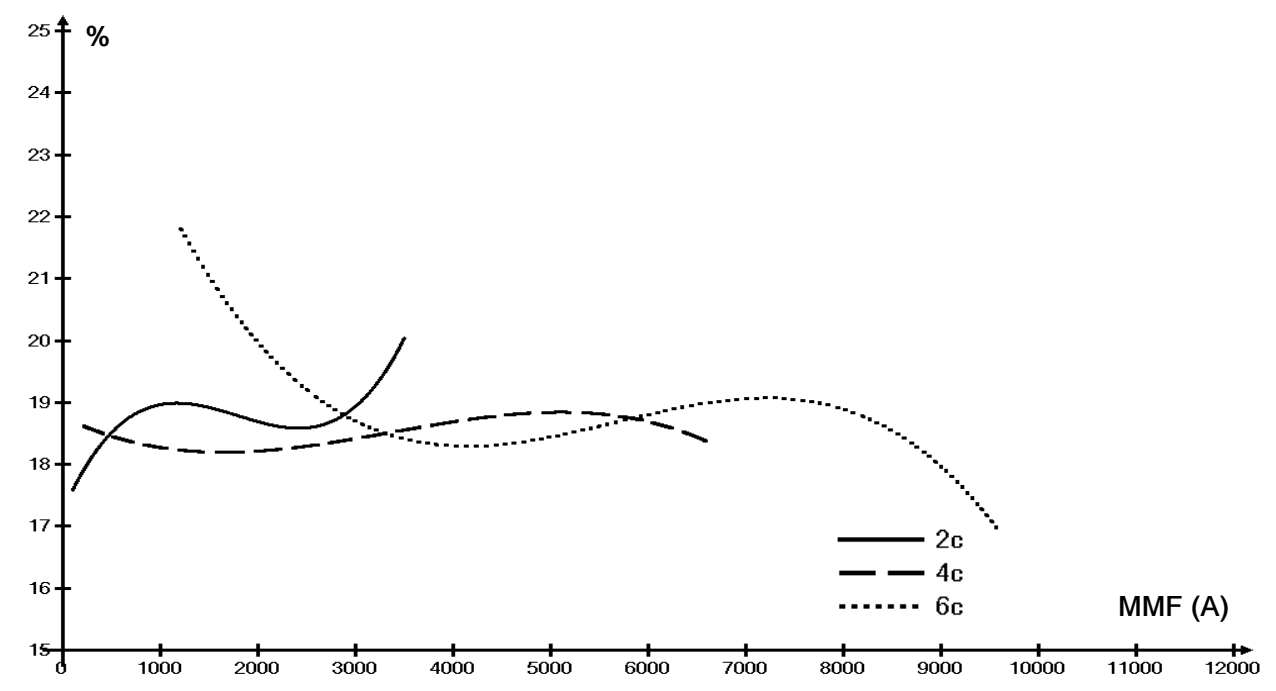

Fig. 8. Configuration 1 with 2, 4 and 6 coils [2]: a) magnetic flux density vs. number of coils, b) magnetic flux density non-uniformity vs. number of coils

Analysis of the experimental results allows one to draw the conclusion that the spatial arrangement of excitation coils does not improve the uniformity of the magnetic field in the air gap in the tested model of EMFG. Better uniformity was obtained for coils located directly on pole, which is in agreement with the known designs of magnetic field generators (Fig. 10). 
a)

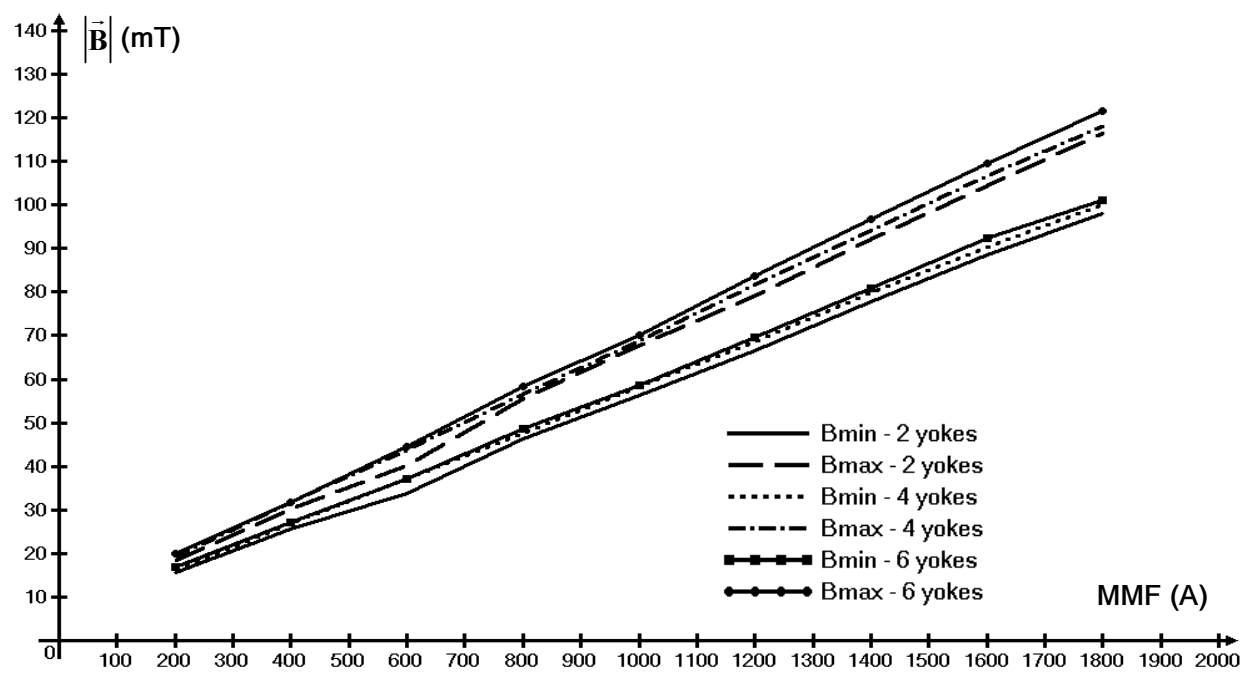

b)

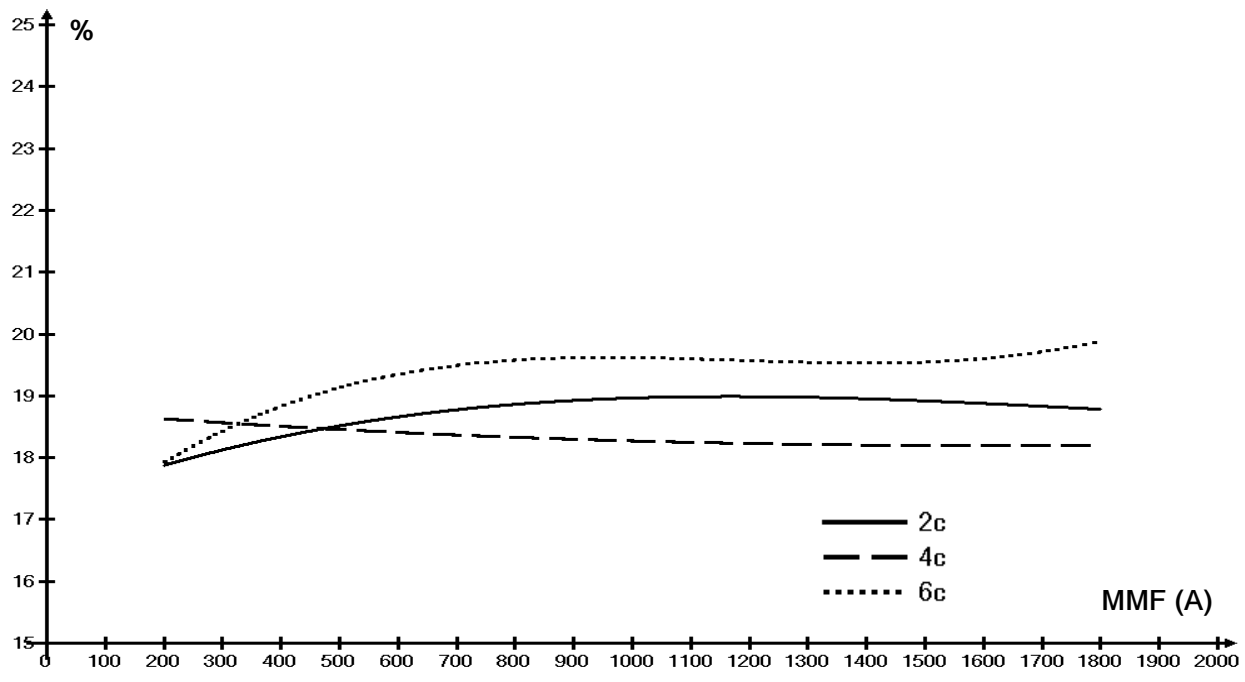

Fig. 9. Configuration 2 with 2, 4 and 6 side yokes [2]: a) magnetic flux density vs. number of side yokes, b) magnetic flux density non-uniformity vs. number of side yokes

The lower the number of coils on the side yokes, the quicker the saturation of the core comes into play (Fig. 8a). For the device structure with two side yokes and two coils on these yokes, the average value of magnetic flux density in the air gap was approximately $40 \mathrm{mT}$.

For configuration with four coils and yokes, the average value of magnetic flux density in the air gap was approximately $65 \mathrm{mT}$. Only the structure with six coils 
and yokes created the possibility to obtain the target value of magnetic flux density $100 \mathrm{mT}$.

a)

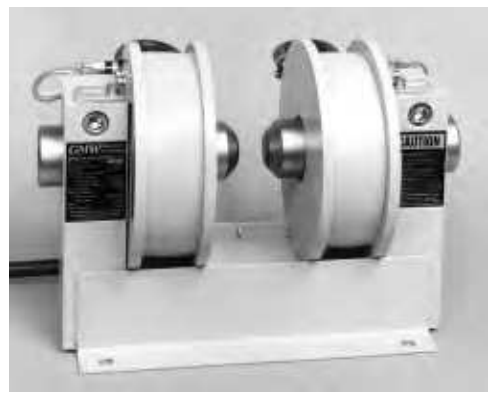

b)

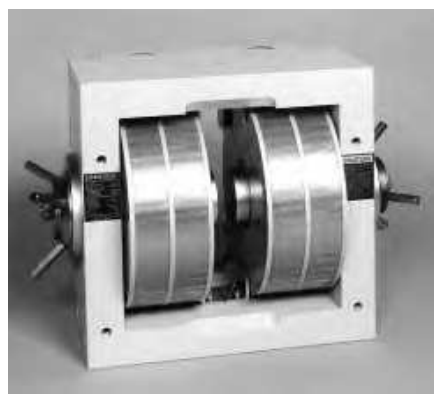

Fig. 10. Magnetic field generator [3]: a) with „C" type of core and two coils on poles, b) with „H” type of core and four coils on poles

The following should be noted from the experimental results. According to initial assumptions, the value of $100 \mathrm{mT}$ should be obtained with MMF of 1884 ampere-turns without the core saturation. For configuration 1, with six coils and yokes, the value of $100 \mathrm{mT}$ is in fact obtained, but with much greater MMF and with the core saturation. For configuration 2 the value of magnetic flux density $100 \mathrm{mT}$ is obtained without the core saturation and with MMF values closer to those given by equation (8). The comparison of magnetic flux density vs. MMF for configuration 1 with six coils and configuration 2 was shown on Figure 11.

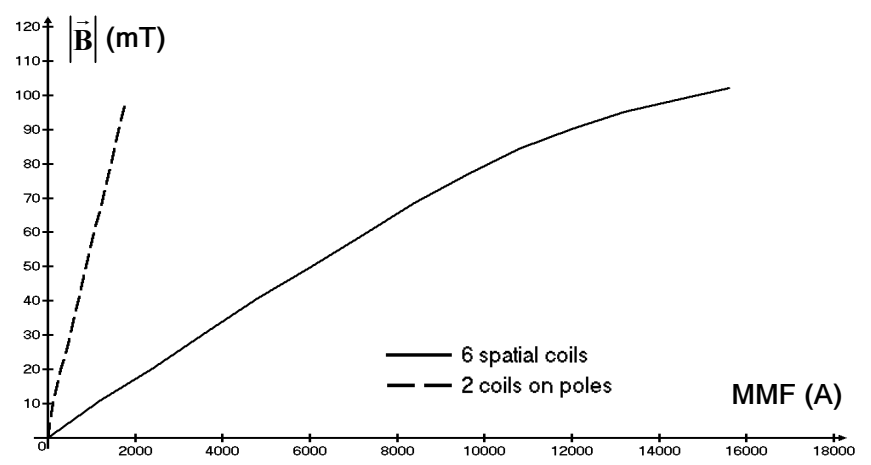

Fig. 11. Comparison of magnetic flux density vs. magnetizing current [2]

The phenomenon of premature core saturation can be explained in a simply way. The premature saturation is caused by the changeable ratio of cross section of yokes to cross section of pole. Taking into consideration cross section of the side yokes and cross section of pole, the ratio is: $0.72: 1$ - for two yokes, 1.44:1 - for four yokes and 2.16:1 - for six yokes. The magnetic fluxes from all yokes are summed in the pole pieces and the formula (e.g. for six yokes with coils) for magnetic flux density takes the form: 


$$
|\vec{B}|=\frac{\Phi_{1}+\Phi_{2}+\Phi_{3}+\Phi_{4}+\Phi_{5}+\Phi_{6}}{S}
$$

where:

$\Phi_{1}=\Phi_{2}=\Phi_{3}=\Phi_{4}=\Phi_{5}=\Phi_{6}=\Phi_{\mathrm{Y}}$,

$S$ - cross section of pole,

$\Phi_{Y}$ - magnetic flux in each yoke.

The highest value of magnetic flux density in the air gap is obtained for six coils on six side yokes - the magnetic flux in pole: $6 \cdot \Phi_{\mathrm{Y}}$ and the lowest magnetic flux density is obtained for two coils on two side yokes - the magnetic flux in pole: $2 \cdot \Phi_{\mathrm{Y}}$. The smaller the number of side yokes with coils, the more the MMF of coils should be increased, to compensate for the inequality $2 \Phi_{\mathrm{Y}}<6 \Phi_{\mathrm{Y}}$ in such a way as to obtain $2 \Phi_{\mathrm{X}}=6 \Phi_{\mathrm{Y}}$ (the magnetic flux $\Phi_{\mathrm{X}}$ should be increased three times in comparison to previous $\Phi_{\mathrm{Y}}$ ). The more the MMF is increased, the higher the flux density in the side yokes leading eventually to the saturation. For this reason different values of magnetic flux density are generated in the air gap for two, four and six side yokes with coils (Fig. 8a). The side yokes are characterized by lower cross section in comparison to the rest part of core, so the saturation firstly affects the yokes.

Considering the configuration 2 , the coils are located on pole pieces, which have larger cross section area than side yokes (the reason why the saturation comes later for them - larger S, lower $|\vec{B}|$ - formula (11)) and the coils generate the magnetic flux, which branches out into smaller fluxes of side yokes, e.g. for six yokes:

$$
\Phi_{I}=\Phi_{I I}=\Phi_{I I I}=\Phi_{I V}=\Phi_{V}=\Phi_{V I}=\Phi / 6
$$

Comparing the two device arrangements, first with coils on pole pieces and two side yokes, and second with two side yokes with two coils, the magnetic flux in side yoke is smaller in the case of the first arrangement. The core of EMFG due to the first arrangement does not suffer from saturation, generating magnetic flux density $100 \mathrm{mT}$ in the air gap (Fig. 9a).

In the case of coils located on side yokes, magnetomotive forces are connected magnetically in parallel and cannot be simply summed. This is similar to an electric circuit with multiple identical parallel voltage sources - the total available voltage is equal to the value of the single voltage source. This can be graphically explained by cutting the hypothetical device into as many pieces as there are side sections. The excitation of each section works on its own cross-section area, but the length of the air gap is the same for each such part, so it requires the same value of MMF, as given by equation (8).

But in the case of coils located on the pole pieces, their magnetomotive forces are connected in series and can be summed. From that point of view such configuration results in much higher electromagnetic efficiency. 


\section{Conclusions}

The test model of magnetic field generator with spatial arrangement of coils made it possible to examine this type of construction, to be used in industrial applications. The modular construction enabled easy and quick device modification. It offers flexibility for various device configurations, which can be an alternative to computer simulations, complicated for 3-D arrangement, especially when magnetic saturation comes into play and accurate material data for mechanically processed parts might be difficult to obtain.

The location of excitation coils around the air gap have no positive influence onto uniformity of magnetic field. Parallel magnetic connection of magnetomotive forces requires energy consumption increased proportionally to the number of section.

From the point of view of supply energy it is better to apply excitation coils on pole pieces, due to better electromagnetic efficiency. Moreover the uniformity of magnetic field is better in the regular device configuration. There is no need for multiple side yokes. They can be limited to two or even one piece, as long as appropriate cross-section area of the magnetic circuit is provided. The construction of a magnetic field generator with spatial coil arrangement can be limited to commonly known and actually used construction (Fig. 10).

The uniformity of magnetic flux in the air gap can be improved in other ways, e.g. using tapered pole pieces [4] or application of magnetic concentrators, but this matter will be a subject of a separate paper.

\section{Acknowledgement}

The paper was realized under the project "Industrial research on actual measurement of energy using 3-phase current transformer modules" co-financed by European Regional Development Fund under the Innovative Economy Operational Programme (Project No. UDA - POIG.01.04.00-24-006/11-00).

\section{References}

[1] Magnetic calculator: www.intemag.com

[2] Influence of the number of excitation coils on uniformity of distribution of magnetic flux density in an air gap. $12^{\text {th }}$ International Workshop on $1 \& 2$ Dimensional Magnetic Measurement and Testing, 3-6 September 2012, Vienna, Austria.

[3] GMW Associates: www.gmw.com

[4] Fiorillo F., Measurement and characterization of magnetic materials, Elsevier, London 2004. 